\title{
Total Knee Arthroplasty- A Prospective Randomised Comparison between Functional Outcomes of Cruciate Retaining and Posterior Stabilising Implants
}

\author{
Pankaj Mahindra ${ }^{1}$, Prabhjeet Singh² ${ }^{2}$ Rajnish Garg ${ }^{3}$, Harpal Singh Selhi' ${ }^{4}$ Maheshinder Singh Chauhan ${ }^{5}$, Ashwani Soni ${ }^{6}$ \\ ${ }^{1}$ Department of Orthopaedics, DMCH, Ludhiana, Punjab, India. ${ }^{2}$ Department of Orthopaedics, SSMMH, Kochi, Kerala, \\ India. ${ }^{3}$ Department of Orthopaedics, DMCH, Ludhiana, Punjab, India. ${ }^{4}$ Department of Orthopaedics, DMCH, Ludhiana, \\ Punjab, India. ${ }^{5}$ Department of Orthopaedics, SPS Hospital, Ludhiana, Punjab, India. ${ }^{6}$ Department of Orthopaedics, \\ GMCH Chandigarh, Punjab, India.
}

\section{ABSTRACT}

\section{BACKGROUND}

Osteoarthritis knee is a leading cause of disability worldwide. Total knee arthroplasty (TKA) is considered as a reliable option to relieve pain and improve lifestyle of patients with osteoarthritis knee. However, there is no single best implant for total knee arthroplasty. Despite being debated since years, the controversy of cruciate retaining (CR) TKA vs Posterior stabilising (PS) TKA is still ongoing. Purpose of our prospective randomised trial is to compare the functional outcomes of CR and PS implants at short term follow-up in patients aged 45 years or more having TKA surgery for OA knee. We hypothesise that there is no difference in outcome of both implants.

\section{METHODS}

All the patients having age 45 years or more admitted to our institute for TKA surgery for OA knee were considered to be included in the study. Patients having knee disease other than OA, history of any knee surgery or high tibial osteotomy were excluded. All knees were randomised and divided into groups- CR and PS. Patients were evaluated preoperatively and at latest follow-up using Knee Society (KS) Knee Score, KS Function Score and the Western Ontario and MacMaster Universities Osteoarthritis Index (WOMAC).

\section{RESULTS}

104 knees in total, were evaluated with average follow-up of $3.9 \pm 1.3$ years in CR group and $3.7 \pm 1.9$ years in PS group. Outcome functional scores WOMAC, KS Knee score and KS Functional score showed significant improvement at final follow-up. However, there is no statistically significant difference among the two groups.

\section{CONCLUSIONS}

Both CR and PS designs are comparable in terms of clinical and functional outcome and implant survivorship at short term follow-up. The choice of design depends upon PCL status and surgeon's preference.

\section{KEY WORDS}

Cruciate Retaining, Posterior Stabilized, Range of Motion, Total Knee Replacement, Functional Outcome, Knee Score
Corresponding Author: Prabhjeet Singh, \#199, Vivekananda Park, Maksudan, Jalandhar, Punjab, India.

E-mail: drgahra@gmail.com

DOI: $10.14260 / \mathrm{jemds} / 2019 / 691$

Financial or Other Competing Interests: None.

How to Cite This Article: Mahindra P, Singh P, Garg R, et al. Total knee arthroplasty- a prospective randomised comparison between functional outcomes of cruciate retaining and posterior stabilising implants. J. Evolution Med. Dent. Sci. 2019;8(43):31853189, DOI: 10.14260/jemds/2019/691

Submission 25-09-2019, Peer Review 22-10-2019,

Acceptance 24-10-2019, Published 28-10-2019. 


\section{BACKGROUND}

Worldwide, osteoarthritis (OA) is estimated to be the leading cause of disability. Earlier, osteoarthritis was known as the most common cause of musculoskeletal pain and disability and affecting mainly elderly population. However, now it is increasingly being diagnosed in younger people in the age group of $35-55$ years. Several reasons can be attributed to this trend globally and in India including growing obesity, sedentary lifestyle, dependence on unhealthy and junk food and vitamin D deficiency. Osteoarthritis is the precipitating diagnosis for more than $90 \%$ of the increasing number of total knee joint replacement operations being undertaken worldwide. Now increasing number of arthroplasties in young population along with increasing life expectancy in operated cases, demands implants and surgeries with more longevity. Total knee arthroplasty (TKA) is considered as a reliable surgical procedure for pain relief in patients with knee osteoarthritis. The standard implant for primary TKA consists of a cemented Cobalt-Chrome femoral component, a polyethylene liner and a titanium tibial baseplate. Some surgeons resurface patella and use polyethylene patellar button. Tibial component can also be a mono-block tibial poly (this significantly reduces cost and has been observed to have equal longevity). There is no debate on importance of correct alignment of components and intra-operative stability of the joint. These factors are of prime importance. Many variations in surgeries had been observed in the past with good outcomes. The two school of thoughts regarding the surgical technique are- "measured resection" and "gap balancing". Both has their own merits. There is also variability in type of implants. From historical prospective, two basic design groups are "anatomic" and "functional". Functional approach had better reproducibility. In functional group, Cruciate sacrificing was first developed (the ICHL and the Total Condylar) followed by Posterior stabilised (PS-TKA) design and the Cruciate-Retaining design (CR-TKA). Some authors believe that CR-TKA has better post-operative knee proprioception and kinaesthesia ${ }^{1,2}$ while others believe that PS TKA has a more reliable femoral rollback, easier ligament balance ${ }^{3,4}$ and better range of motion (ROM). ${ }^{5}$

The PS implant is designed with the goal of mechanical alignment where tibial and femoral cuts are made perpendicular to the mechanical axis. PS knees are often easier to balance as compared to CR knees because of lesser number of ligaments to be balanced. On the other hand, CR design uses native PCL to prevent anterior dislocation of the femur during knee flexion. So, there is no cam-post mechanism and therefore no need for box cut and hence it preserves bone. The main rationale behind CR knee design is that PCL is critical to normal knee kinematics.

However, the recent reviews and meta-analysis did not find any significant difference in knee scores, radiological outcomes and complications between PS and CR knee replacement.6-8 Despite being debated since years, the controversy of CR total knee arthroplasty (TKA) vs PS TKA is still ongoing. In our prospective randomised trial, we compared the functional outcomes of CR and PS implants at short term follow-up in patients age 45 years or more having TKA surgery for OA knee. We hypothesise that there is no difference in outcome of both implants.

\section{METHODS}

This is a prospective randomized trial study. All the patients having age 45 years and more admitted to our institute for TKA surgery for OA knee were considered to be included in the study. It is assumed that patient admission for total knee replacement surgery in our hospital has prevalence rate of 7.5\% based on previous data. Assumptions: Confidence Level $=95 \%$ Precision $(\mathrm{d})= \pm 5 \%$. For estimation of sample size, the following formula has been used $n=\left(\mathrm{Z}^{2} \alpha\right.$ X P X (1-P) $) / \mathrm{d}^{2}$ where; $\mathrm{Z} \alpha=$ Value of standard normal variate corresponding to $\alpha$ level of significance [P = Likely value of parameter, $\mathrm{Q}=1$ $\mathrm{P}, \mathrm{D} .=$ margin of errors which is a measure of precision]. With these assumptions the sample size works out as 106 i.e. 53 each group. One patient in the PS group where bilateral knee replacement was done, was lost to follow up. So final data is based upon 53 knees in CR group whereas 51 knees in PS group. Written informed consent was taken from patients before enrolling them in study. Patients having knee disease other than OA were excluded. Patients with history of any knee surgery or high tibial osteotomy were excluded. All knees were randomised at the time of admission using computer generated charts and were divided into two groups. Group CR for CR implants and group PS for PS implants. After getting all the clearances for surgery, TKA was done by standard parapatellar approach in all cases. Surgery with intramedullary rod for femoral cuts, extramedullary guide for tibial cuts, and spacer block for appropriate ligament balancing was performed in all patients. Patella was resurfaced in all patients and all implants were fixed with cement.

A well-informed written consent was taken from all the patients before including them in study and all the patients were explained about the type of implants used. Postoperatively patients underwent a standard rehabilitation program consisting of early ROM and weight-bearing exercises as tolerated. All patients received prophylactic anticoagulants and antibiotics as necessary. Patients were evaluated preoperatively and at latest follow-up using Knee Society (KS) Knee Score, KS Function Score and the Western Ontario and MacMaster Universities Osteoarthritis Index (WOMAC). ${ }^{9,10,11}$ The study was approved by ethical board of our institute.

\section{Statistical Analysis}

Statistical analysis was done using software Statistical Package for the Social Sciences (SPSS). Kolmogorov-Smirnov test was used to determine normal distribution of data. Continuous variables were compared using Student T test. Paired T-test was used to compare preoperative data with latest follow-up. $\mathrm{p}$-Value was determined and value less than 0.05 was considered significant. 


\section{RESULTS}

\section{Demographics (Table 1)}

Total 104 knees in 68 patients were included in the study who underwent TKA from December 2013 to May 2015. 36 patients underwent bilateral and 32 patients underwent unilateral TKA. Mean age in CR group was 60 years and 62 years in PS group. In group CR 17 patients underwent bilateral CR TKA and 12 patients underwent unilateral CR TKA. In group PS 12 patients underwent bilateral PS TKA and 20 patients underwent unilateral PS TKA. In 7 patients CR TKA was done on one side and PS TKA was done on other side. These 7 patients were included in both groups while doing demographic evaluation.

\section{Preoperative Parameters (Table 2)}

Both groups had preoperative varus malalignment. Mean ROM was slightly better in PS group; however, it was not statistically significant. Both groups had poor knee functional outcome scores preoperatively and the difference among two groups was not statistically significant.

\section{Outcome Measures (Table 3)}

Average follow-up was 3.9 years in CR group and 3.7 years in PS group. Mean ROM is better in PS group at 5 months and at final follow-up, but the difference was not significant statistically. Also, there was no statistically significant difference among two groups at 5 months or final follow-up in view of tibia-femoral angle, KS Knee score and KS Functional score. There was no revision surgery in any group till last follow-up.

\begin{tabular}{|c|c|c|}
\hline & Group CR (n=36) & Group PS (n=39) \\
\hline Mean Age (Years) & 60 & 62 \\
\hline Bilateral TKA & 17 & 12 \\
\hline Unilateral TKA & 12 & 20 \\
\hline Mix TKA & 7 & 7 \\
\hline Male: female & $6: 30$ & $13: 26$ \\
\hline \multicolumn{2}{|c|}{ Table 1. Demographic Profile of Patients } \\
\hline
\end{tabular}

\begin{tabular}{|c|c|c|c|}
\hline & $\begin{array}{c}\text { Group CR } \\
(\mathbf{n = 5 3 )}\end{array}$ & $\begin{array}{c}\text { Group PS } \\
(\mathbf{n = 5 1})\end{array}$ & $\mathbf{p}$ \\
\hline Tibio-femoral angle(varus) & $6.85 \pm 2.48$ & $6.39 \pm 2.96$ & 0.395 \\
\hline ROM & $89.23 \pm 18.57$ & $94.61 \pm 10.67$ & 0.074 \\
\hline WOMAC score & $67.45 \pm 8.23$ & $70.25 \pm 4.89$ & 0.058 \\
\hline KS knee score & $45.8 \pm 4.1$ & $46.5 \pm 5.1$ & 0.446 \\
\hline KS function score & $35.8 \pm 7.9$ & $34.6 \pm 8.4$ & 0.565 \\
\hline \multicolumn{2}{|c|}{ Table 2. Pre-Operative Clinical Evaluation of 104 Knees } \\
\hline
\end{tabular}

\begin{tabular}{|c|c|c|c|}
\hline & Group CR (n=53) & Group PS (n=51) & p \\
\hline Final Follow-up (Mean years) & $3.9 \pm 1.3$ & $3.7 \pm 1.9$ & 0.245 \\
\hline \multicolumn{4}{|c|}{ Tibio-Femoral Angle } \\
\hline (5 mth follow-up) & $2.04 \pm 0.78$ & $2.53 \pm 0.78$ & 0.214 \\
\hline (final follow-up) & $2.1 \pm 0.71$ & $2.6 \pm 0.67$ & 0.208 \\
\hline \multicolumn{4}{|c|}{ ROM } \\
\hline (5 mth follow-up) & $118 \pm 16.79$ & $122 \pm 9.29$ & 0.206 \\
\hline (final follow-up) & $117 \pm 15.62$ & $120 \pm 10.34$ & 0.432 \\
\hline WOMAC score & $5.92 \pm 1.86$ & $7.45 \pm 2.56$ & 0.214 \\
\hline (5 mth follow-up) & $6.24 \pm 1.92$ & $7.52 \pm 1.96$ & 0.312 \\
\hline (final follow-up) & $90.5 \pm 6.4$ & $89.5 \pm 7.8$ & 0.768 \\
\hline KS knee score & $91.4 \pm 7.8$ & $92.6 \pm 7.5$ & 0.684 \\
\hline (5 mth follow-up) & $85.7 \pm 6.5$ & $87.6 \pm 6.8$ & 0.257 \\
\hline (final follow-up) & $84.6 \pm 7.2$ & $88.6 \pm 8.4$ & 0.723 \\
\hline \multicolumn{4}{|c|}{ KS Function Score } \\
\hline (5 mth follow-up) & (final follow-up) & Table 3. Follow-Up Clinical Evaluation of 104 Knees \\
\hline \multicolumn{4}{|c|}{} \\
\hline
\end{tabular}

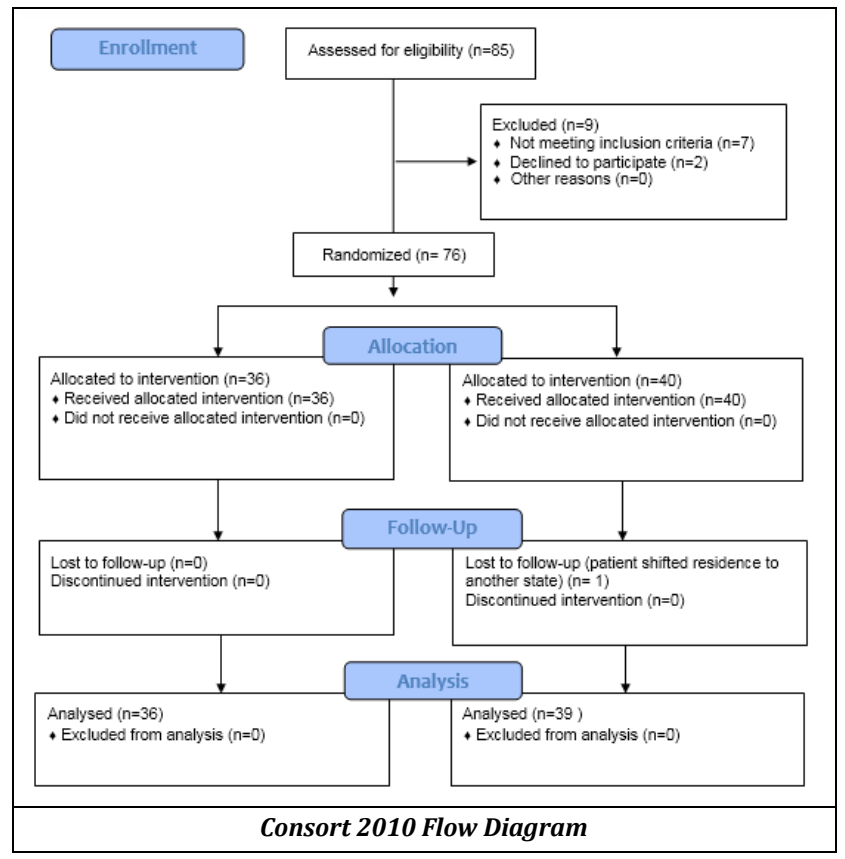

DISCUSSION

Pain relief, restoration of knee kinematics, function and longterm implant survivorship are the desirable outcomes of successful knee arthroplasty. ${ }^{12}$ Multiple designs have been devised till date for achieving above said outcomes. However, CR and PS remain commonly used designs in present time. ${ }^{13}$ The superiority of one design over another is still matter of ongoing discussion. Numerous studies have been done till date in order to compare functional or biomechanical superiority of these designs (Table 4).14 Most of the studies have small sample size and laid emphasis on radiological, kinematics and short-term functional analysis. Both type of prosthesis has been proven safe and reliable in the treatment of severe arthritis. Both designs are comparable in terms of functional outcome and complications and there were no statistically significant differences. ${ }^{15,16}$

Merits of CR design includes better restoration of knee kinematics similar to native knee, enhanced femoral rollback during flexion, greater articular stability, proprioception, thin bone cuts and less blood loss ${ }^{17-19}$. Demerits include high polyethylene wear as femoral rollback in absence of intact anterior cruciate ligament is mere combination of rolling and sliding leading to generation of high contact stresses. ${ }^{20}$

PS design is recommended in inflammatory conditions leading to PCL degeneration and in cases with chronic PCL pathology. Advantages include easy technique, more comfortable soft tissue balancing, enhanced stability, better (ROM) and patella-femoral kinematics. ${ }^{21,22}$ Demerits include patellar clunk and increased wear due to post. ${ }^{23,24}$

Alignment and ROM- In our study, there was marked improvement in tibio-femoral angle from preoperative values. However, there was no difference among the groups and both groups had slight valgus alignment at final follow-up. There was slight increase in ROM in PS group at final follow-up, but that difference was not statistically significant. Berick et al reported statistically significant greater knee flexion and (ROM) with PS design, however clinically irrelevant ${ }^{7}$. 


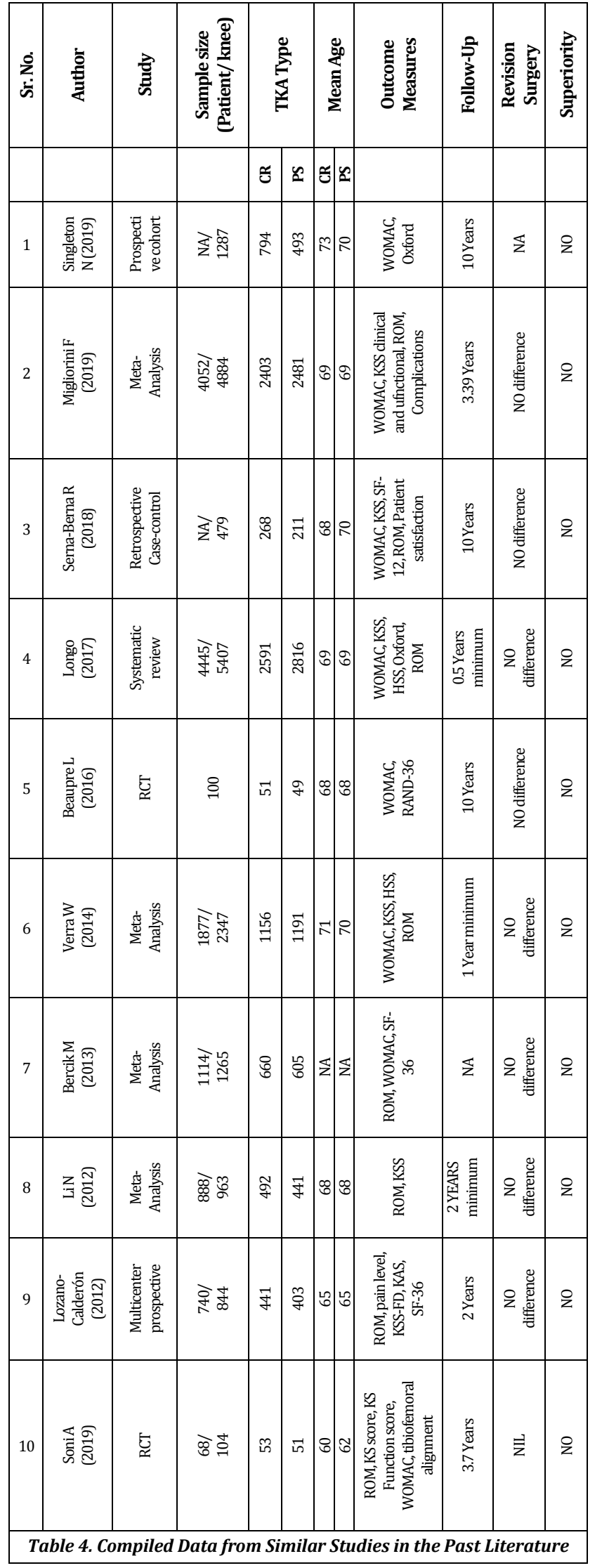

\section{Knee Function}

Knee function was evaluated using validated score like WOMAC and KSS Clinical and Functional. There was no statistically significant difference among CR and PS groups at 5 months and at final follow-up. The findings of our study are in total agreement with the recent literature. The functional outcomes reported in recent studies have been mentioned in Table 4. For determining functional outcome, choice of scoring is very essential. Both WOMAC and Oxford knee score mentioned in various studies are validated scores. ${ }^{25,26}$ However prime consideration should be given to minimally clinical important difference (MCID). ${ }^{27}$ It refers to quantification of minimal clinical difference, a patient and surgeon can appreciate with respect to scoring method. Younger patients having high functional demands undergoing TKA should be evaluated with a new score high activity arthroplasty score (HAAS). ${ }^{28}$

\section{Implant Survivorship}

Most of the studies have shorter follow-up period than our study. We found only two recent studies having longer followup period. ${ }^{10,16}$ There was no difference in longevity of these two designs at 10 years follow-up. There was no revision surgery in our study till last follow-up. On the positive side, our study is a prospective randomized trial and having adequate sample size. We used different manufacture designs so our results can be generalized to the commonly used CR and PS designs. The limitation of our study is a short follow-up period. Although there was no revision surgery, a longer follow-up is necessary for predicting implant longevity. However, more research is required to be done in younger age group patients (Between 45 -60 years) as knee arthroplasty is increasingly being done in this age group owing to increase incidence of obesity, leading to early arthritis. Further high quality RCTs may be needed for evaluating stair climbing ability.

\section{CONCLUSIONS}

Both CR and PS designs are comparable in terms of clinical and functional outcome and implant survivorship at short term follow-up. The choice of design depends upon PCL status and surgeon's preference.

\section{REFERENCES}

[1] Nelissen RG, Hogendoorn PC. Retain or sacrifice the posterior cruciate ligament in total knee arthroplasty? A histopathological study of the cruciate ligament in osteoarthritic and rheumatoid disease. J Clin Pathol 2001;54(5):381-4.

[2] Swanik CB, Lephart SM, Rubash HE. Proprioception, kinesthesia and balance after total knee arthroplasty with cruciate-retaining and posterior stabilized prostheses. J Bone Joint Surg Am 2004;86(2):328-34.

[3] Conditt MA, Noble PC, Bertolusso R, et al. The PCL significantly affects the functional outcome of total knee arthroplasty. J Arthroplasty 2004;19(7 Suppl 2):107-12.

[4] Straw R, Kulkarni S, Attfield S, et al. Posterior cruciate ligament at total knee replacement. Essential, beneficial or a hindrance? J Bone Joint Surg Br 2003;85(5):671-4.

[5] Harato K, Bourne RB, Victor J, et al. Midterm comparison of posterior cruciate retaining versus -substituting total knee arthroplasty using the Genesis II prosthesis. A multicenter prospective randomized clinical trial. Knee 2008;15:217-21. 
[6] Luo SX, Zhao JM, Su W, et al. Posterior cruciate substituting versus posterior cruciate retaining total knee arthroplasty prostheses: a meta-analysis. Knee 2012;19(4):246-52.

[7] Bercik MJ, Joshi A, Parvizi J. Posterior cruciate-retaining versus posterior-stabilized total knee arthroplasty: a meta-analysis. J Arthroplasty 2013;28(3):439-44.

[8] Li N, Tan Y, Deng Y, et al. Posterior cruciate-retaining versus posterior stabilized total knee arthroplasty: a meta-analysis of randomized controlled trials. Knee Surg Sports Traumatol Arthrosc 2014;22(3):556-64.

[9] Collins NJ, Misra D, Felson DT, et al. Measures of knee function: International Knee Documentation Committee (IKDC) Subjective Knee Evaluation Form, Knee Injury and Osteoarthritis Outcome Score (KOOS), Knee Injury and Osteoarthritis Outcome Score Physical Function Short Form (KOOS-PS), Knee Ou. Arthritis Care \& Research 2011;(63 Suppl 11):S208-S28.

[10] Liow RY, Walker K, Wajid MA, et al. The reliability of the American Knee Society Score. Acta Orthopaedica Scandinavica 2000;71(6):603-8.

[11] Medalla GA, Moonot P, Peel T, et al. Cost-benefit comparison of the Oxford Knee Score and the American Knee Society Score in measuring outcome of total knee arthroplasty. The Journal of Arthroplasty 2009;24(4):652-6.

[12] Singleton N, Nicholas B, Gormack N, et al. Differences in outcome after cruciate retaining and posterior stabilized total knee arthroplasty. J Orthop Surg 2019;27(2):2309499019848154.

[13] Serna-Berna R, Lizaur-Utrilla A, Vizcaya-Moreno MF, et al. Cruciate-retaining vs posterior-stabilized primary total arthroplasty. Clinical outcome comparison with a minimum follow-up of 10 years. J Arthroplasty 2018;33(8):2491-5.

[14] Verra WC, Boom LG, Jacobs WC, et al. Similar outcome after retention or sacrifice of the posterior cruciate ligament in total knee arthroplasty. Acta Orthop 2015;86(2):195-201.

[15] Migliorini F, Eschweiler J, Tingart M, et al. Posteriorstabilized versus cruciate-retained implants for total knee arthroplasty: a meta-analysis of clinical trials. Eur J Orthop Surg Traumatol 2019;29(4):937-46.

[16] Lozano-Calderón SA, Shen J, Doumato DF, et al. Cruciateretaining vs posterior-substituting inserts in total knee arthroplasty: functional outcome comparison. J Arthroplasty 2013;28(2):234-42.e1.
[17] Hunt NC, Ghosh KM, Blain AP, et al. No statistically significant kinematic difference found between a cruciate-retaining and posterior-stabilized Triathlon knee arthroplasty: a laboratory study involving eight cadavers examining soft-tissue laxity. Bone Joint J 2015;97-B(5):642-8.

[18] Matsumoto T, Kubo S, Muratsu H, et al. Different pattern in gap balancing between the cruciate-retaining and posterior-stabilized total knee arthroplasty. Knee Surg Sports Traumatol Arthrosc 2013;21(10):2338-45.

[19] Beaupre LA, Sharifi B, Johnston DWC. A randomized clinical trial comparing posterior cruciate-stabilizingvs posterior cruciate-retaining prostheses in primary total knee arthroplasty: 10-year follow-up. J Arthroplasty 2017;32(3):818-23.

[20] Naudie DD, Ammeen DJ, Engh GA, et al. Wear and osteolysis around total knee arthroplasty. J Am Acad Orthop Surg 2007;15(1):53-64.

[21] Ritter MA, Berend ME, Meding JB, et al. Long-term followup of anatomic graduated components posterior cruciate-retaining total knee replacement. Clin Orthop Relat Res 2001;(388):51-7.

[22] Becher C, Heyse TJ, Kron N, et al. Posterior stabilized TKA reduce patellofemoral contact pressure compared with cruciate retaining TKA in vitro. Knee Surg Sports Traumatol Arthrosc 2009;17(10):1159-65.

[23] Callaghan JJ, O’Rourke MR, Goetz DD, et al. Tibial post impingement in posterior-stabilized total knee arthroplasty. Clin Orthop Relat Res 2002;(404):83-8.

[24] Hozack WJ, Rothman RH, Booth RE Jr, et al. The patellar clunk syndrome. Clin Orthop Relat Res 1989;(241):203-8.

[25] Thumboo J, Chew LH, Soh CH. Validation of the Western Ontario and McMaster University Osteoarthritis Index in Asians with Osteoarthritis in Singapore. Osteoarthritis Cartilage 2001;9(5):440-6.

[26] Conaghan PG, Emerton M, Tennant A. Internal construct validity of the Oxford Knee Scale: evidence from Rasch measurement. Arthritis Rheum 2007;57(8):1363-7.

[27] Angst F, Aeschlimann A, Stucki G. Smallest detectable and minimally clinically important differences of rehabilitation intervention with their implications for required sample sizes using WOMAC and SF-36 quality of life measurement instruments in patients with osteoarthritis of the lower extremities. Arthritis Rheum 2001;45(4):384-91.

[28] Talbot S, Hooper G, Stokes A, et al. Use of a new high activity arthroplasty score to assess function of young patients with total hip or knee arthroplasty. J Arthroplasty 2010;25(2):268-73. 